\title{
Warfarin and Aspirin in Patients with Heart Failure and Sinus Rhythm
}

\author{
Shunichi Homma, M.D., John L.P. Thompson, Ph.D., Patrick M. Pullicino, M.D., Bruce \\ Levin, Ph.D., Ronald S. Freudenberger, M.D., John R. Teerlink, M.D., Susan E. Ammon, \\ N.P., Susan Graham, M.D., Ralph L. Sacco, M.D., Douglas L. Mann, M.D., J.P. Mohr, M.D., \\ Barry M. Massie, M.D., Arthur J. Labovitz, M.D., Stefan D. Anker, M.D., Ph.D., Dirk J. Lok, \\ M.D., Piotr Ponikowski, M.D., Ph.D., Conrado J. Estol, M.D., Ph.D., Gregory Y.H. Lip, M.D., \\ Marco R. Di Tullio, M.D., Alexandra R. Sanford, M.S., Vilma Mejia, B.S., Andre P. Gabriel, \\ M.D., Mirna L. del Valle, B.S., Richard Buchsbaum, and for the WARCEF Investigators* \\ Columbia University Medical Center, New York (S.H., J.L.P.T., B.L., J.P.M., M.R.D.T., V.M., \\ A.P.G., M.L.V., R.B., A.R.S.); the University of Kent, Canterbury (P.M.P.), and the University of \\ Birmingham Centre for Cardiovascular Sciences, Birmingham (G.Y.H.L.) - both in the United \\ Kingdom; Lehigh Valley Hospital, Allentown, PA (R.S.F.); the University of California, San \\ Francisco (J.R.T., B.M.M.), and San Francisco VA Medical Center (J.R.T., B.M.M., S.E.A.) - \\ both in San Francisco; the State University of New York at Buffalo, Buffalo (S.G.); the University \\ of Miami, Miami (R.L.S.); Washington University, St. Louis (D.L.M.); the University of South \\ Florida, Tampa (A.J.L.); Charité Universitätsmedicin, Campus Virchow-Klinikum, Berlin (S.D.A.); \\ the Center for Clinical and Basic Research, IRCCS San Raffaele, Rome (S.D.A.); Hospital \\ Deventer, Deventer, the Netherlands (D.J.L.); Military Hospital, Wroclaw, Poland (P.P.); and \\ Centro Neurológico de Tratamiento y Rehabilitación, Buenos Aires (C.J.E.).
}

\section{Abstract}

BACKGROUND—It is unknown whether warfarin or aspirin therapy is superior for patients with heart failure who are in sinus rhythm.

METHODS-We designed this trial to determine whether warfarin (with a target international normalized ratio of 2.0 to 3.5) or aspirin (at a dose of $325 \mathrm{mg}$ per day) is a better treatment for patients in sinus rhythm who have a reduced left ventricular ejection fraction (LVEF). We followed 2305 patients for up to 6 years (mean $[ \pm \mathrm{SD}], 3.5 \pm 1.8$ ). The primary outcome was the time to the first event in a composite end point of ischemic stroke, intracerebral hemorrhage, or death from any cause.

RESULTS-The rates of the primary outcome were 7.47 events per 100 patient-years in the warfarin group and 7.93 in the aspirin group (hazard ratio with warfarin, $0.93 ; 95 \%$ confidence interval $[\mathrm{CI}], 0.79$ to $1.10 ; \mathrm{P}=0.40$ ). Thus, there was no significant overall difference between the two treatments. In a time-varying analysis, the hazard ratio changed over time, slightly favoring warfarin over aspirin by the fourth year of follow-up, but this finding was only marginally significant $(\mathrm{P}=0.046)$. Warfarin, as compared with aspirin, was associated with a significant

Copyright $@ 2012$ Massachusetts Medical Society.

Address reprint requests to: Dr. Homma at Columbia University Medical Center, PH 3-342, 622 West 168th St., New York, NY 10032.

Drs. Homma and Thompson contributed equally to this article.

*The investigators in the Warfarin versus Aspirin in Reduced Cardiac Ejection Fraction (WARCEF) Study Group are listed in the Supplementary Appendix, available at NEJM.org.

No other potential conflict of interest relevant to this article was reported.

Disclosure forms provided by the authors are available with the full text of this article at NEJM.org. 
reduction in the rate of ischemic stroke throughout the follow-up period ( 0.72 events per 100 patient-years vs. 1.36 per 100 patient-years; hazard ratio, $0.52 ; 95 \% \mathrm{CI}, 0.33$ to $0.82 ; \mathrm{P}=0.005$ ). The rate of major hemorrhage was 1.78 events per 100 patient-years in the warfarin group as compared with 0.87 in the aspirin group $(\mathrm{P}<0.001)$. The rates of intracerebral and intracranial hemorrhage did not differ significantly between the two treatment groups $(0.27$ events per 100 patient-years with warfarin and 0.22 with aspirin, $\mathrm{P}=0.82$ ).

CONCLUSIONS-Among patients with reduced LVEF who were in sinus rhythm, there was no significant overall difference in the primary outcome between treatment with warfarin and treatment with aspirin. A reduced risk of ischemic stroke with warfarin was offset by an increased risk of major hemorrhage. The choice between warfarin and aspirin should be individualized.

Chronic heart failure is a major cause of illness and death. Heart failure is associated with a hypercoagulable state, formation of left ventricular thrombus, and cerebral embolism. ${ }^{1,2}$ It is also associated with both sudden death and death resulting from progressive heart failure that may be caused by unrecognized atherothrombotic events. ${ }^{3}$ As a result, there is a rationale for using oral anticoagulants to treat patients with chronic heart failure who are in sinus rhythm. However, the role of oral anticoagulants as compared with aspirin has not been clarified in patients with chronic heart failure. ${ }^{4-6}$ Early studies showed that anticoagulation reduced the rates of embolic events and death, but many patients in these trials had atrial fibrillation and clinically significant valvular heart disease, making interpretation of the results difficult. ${ }^{7-9}$ In retrospective analyses of data from large trials involving patients with a reduced left ventricular ejection fraction (LVEF), conflicting results have been reported. ${ }^{10-13}$ Unfortunately, these findings are of limited value, since the use of anticoagulants was not randomized or controlled, data were collected retrospectively, end points were not predefined or standardized, and patients with atrial fibrillation were included.

Several prospective studies comparing oral anticoagulants with aspirin were too small to provide conclusive evidence for the superiority of either agent. ${ }^{14-16}$ In the Heart Failure Long-Term Antithrombotic Study (HELAS), 197 patients were randomly assigned to warfarin, aspirin, or placebo; there was no significant difference among the groups in the incidence of embolic events. ${ }^{14}$ In the Warfarin/Aspirin Study in Heart Failure (WASH), 279 patients were randomly assigned to warfarin, aspirin, or placebo; there was no significant difference among the groups in the composite end point of death, stroke, or myocardial infarction, but the rate of hospitalization was highest among those receiving aspirin. ${ }^{15}$ The Warfarin and Antiplatelet Therapy in Chronic Heart Failure trial (WATCH), which was the most recent and the largest study, enrolled 1587 patients who were randomly assigned to warfarin, aspirin, or clopidogrel, with a mean follow-up period of 1.9 years. ${ }^{16}$ The results of this trial, which was terminated prematurely owing to difficulties with recruitment, suggested that there was a reduction in the rate of ischemic stroke with warfarin as compared with aspirin but showed an increase in hospitalization for heart failure in the aspirin group as compared with the warfarin group. The Warfarin versus Aspirin in Reduced Cardiac Ejection Fraction (WARCEF) trial was designed to compare the efficacy and safety of warfarin with those of aspirin among a substantially larger number of patients, with the use of a double-blind, randomized design. ${ }^{17}$

\section{METHODS STUDY DESIGN AND OVERSIGHT}

We conducted a cooperative, double-blind, multicenter clinical trial at 168 centers in 11 countries. The trial was sponsored by the National Institutes of Health (NIH), with an independently funded clinical coordinating center and statistical analysis center. Warfarin 
and warfarin placebo were provided by Taro Pharmaceuticals U.S.A., and aspirin and aspirin placebo by Bayer HealthCare. Neither of these companies had any role in the design of the study, the collection or analysis of the data, the writing of the manuscript, or the decision to submit the manuscript for publication. The target international normalized ratio (INR) was 2.75 , with an acceptable target range of 2.0 to 3.5. To minimize variations in blood processing, blood samples for determination of the INR were processed at selected central laboratories. To confirm the accuracy of LVEF assessment, personnel at two core echocardiography laboratories (in St. Louis and New York) who were unaware of the treatment assignments reviewed the echocardiographic studies. An independent end-point adjudication committee, whose members were unaware of the treatment assignments, adjudicated all primary and secondary outcomes and major hemorrhages. The trial protocol, available with the full text of this article at NEJM.org, was approved by the institutional review board at each participating center. The first two authors assume responsibility for the completeness and accuracy of the data and analyses and for the fidelity of the study to the protocol. Written informed consent was obtained from each patient. Patient recruitment started in October 2002 and ended in January 2010. The maximum follow-up time was 6 years, and the minimum was 1 year. An independent data and safety monitoring board appointed by the NIH monitored trial operations.

\section{STUDY PATIENTS}

Eligible patients were 18 years of age or older and had normal sinus rhythm, no contraindication to warfarin therapy, and an LVEF of 35\% or less as assessed by

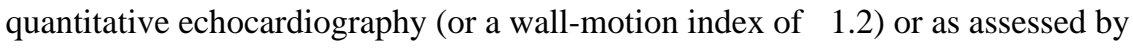
radionuclide or contrast ventriculography within 3 months before randomization. Patients who had a clear indication for warfarin or aspirin were not eligible. Patients in any New York Heart Association (NYHA) functional class were eligible, but patients in NYHA class I could account for no more than $20 \%$ of the total number of patients undergoing randomization. Additional eligibility criteria were a modified Rankin score of 4 or less (on a scale of 0 to 6 , with higher scores indicating more severe disability), and planned treatment with a beta-blocker, an angiotensin-converting-enzyme (ACE) inhibitor (or, if the sideeffect profile with ACE inhibitors was unacceptable, with an angiotensin-receptor blocker), or hydralazine and nitrates. Patients were ineligible if they had a condition that conferred a high risk of cardiac embolism, such as atrial fibrillation, a mechanical cardiac valve, endocarditis, or an intracardiac mobile or pedunculated thrombus.

\section{STUDY MEDICATION}

In the double-blind, double-dummy design, patients who were assigned to active warfarin received warfarin and placebo aspirin, and patients assigned to active aspirin received aspirin and placebo warfarin. ${ }^{18}$ The statistical analysis center fabricated clinically plausible INR results for patients in the aspirin group and provided these results to the sites, along with the actual INR results for the patients in the warfarin group, so that all the patients were treated as if they were receiving active warfarin.

\section{FOLLOW-UP}

Follow-up was performed monthly by telephone or in person at the time blood was obtained for determination of the INR, to assess adherence to the study drug and to regulate INR values. A followup assessment in person was also conducted quarterly for a clinical evaluation and annually for a detailed examination. All data were entered into the Webbased communications interface that was developed and managed by the statistical analysis center. 


\section{ASSESSMENT OF OUTCOMES AND MAJOR ADVERSE EVENTS}

The primary outcome was the time to the first event in a composite end point of ischemic stroke, intracerebral hemorrhage, or death from any cause. Stroke was defined as a clinically relevant new lesion detected on computed tomography or magnetic resonance imaging (MRI) or, in the absence of a new lesion, clinical findings that were consistent with the occurrence of clinical stroke and that lasted for longer than 24 hours. The main secondary outcome was the first event in a composite of the primary outcome, myocardial infarction, or hospitalization for heart failure. Major hemorrhage was defined as intracerebral, epidural, subdural, subarachnoid, spinal intramedullary, or retinal hemorrhage; any other bleeding causing a decline in the hemoglobin level of more than $2 \mathrm{~g}$ per deciliter in 48 hours; or bleeding requiring transfusion of 2 or more units of whole blood, hospitalization, or surgical intervention. Minor hemorrhage was defined as any nonmajor hemorrhage.

\section{STATISTICAL ANALYSIS}

The primary null hypothesis was that the time to the first event in the composite primary end point (ischemic stroke, intracerebral hemorrhage, or death from any cause) would not differ significantly between the group receiving warfarin therapy and the group receiving aspirin therapy. The main secondary null hypothesis was that the time to the first event of the primary outcome, myocardial infarction, or hospitalization for heart failure would not differ significantly between the two groups.

The original target sample size was 2860 patients, providing $89 \%$ power to test the primary null hypothesis in the intention-to-treat population, with the use of a log-rank test and a twosided probability of a type I error of 5\%, assuming a hazard rate reduction of $17.82 \%$ in either group as compared with the other, after adjustment for use or nonuse of beta-blockers and allowance for discontinuation of therapy, dropout, and crossover (e.g., owing to the development of atrial fibrillation). In 2009, because of slow recruitment, a plan was developed to stop recruitment in 2010 and to extend the maximum follow-up time from 5 years to 6 years, resulting in a projected sample size of 2303 and power of approximately $65 \%$. The final sample of 2305 patients yielded a sufficient number of outcomes for the study to have $69 \%$ power to test the primary null hypothesis and $83 \%$ power for the main secondary null hypothesis.

Both major study hypotheses were prespecified and were tested according to the intentionto-treat principle at a two-tailed alpha level of 0.05 . For the test of the primary null hypothesis, the statistical analysis plan prespecified the use of a log-rank test to compare the cumulative incidence curves in the treatment groups if log-minus-log survival curves did not show a violation of the proportional-hazards assumption and a Cox model with time-varying hazard ratios if they did. Since the log-minus-log survival curves crossed, we report the results of the log-rank test as the primary analysis and, secondarily, the results from the Cox model, which expresses the log-relative hazard ratio as a linear function of follow-up time. A pre-specified interim monitoring procedure was performed according to the method of Haybittle and Peto, with conservative stopping boundaries for the interim analyses of logrank z scores in excess of \pm 3.2905 (corresponding to a nominal two-tailed $P$ value of 0.001 ). Because very little type I error was spent with this procedure, we report only the unadjusted $\mathrm{P}$ values. Hazard ratios for individual components of the outcomes were obtained from cause-specific proportional-hazards models with or without time-varying coefficients, depending on their statistical significance at an alpha level of 0.05 . To help weigh overall risks and benefits, we conducted a post hoc safety analysis that added intracranial hemorrhage to the components of the primary outcome. 


\section{RESULTS \\ STUDY PATIENTS}

From October 2002 through January 2010, a total of 2305 patients were enrolled (1119 in the United States and Canada and 1186 in Europe and Argentina). The mean [ \pm SD] followup time was $3.5 \pm 1.8$ years, and the total follow-up time was 8225 patient-years. The clinical and demographic characteristics of the patients are shown in Table 1. Survival status was known for $97.0 \%$ of the patients. A total of 34 patients (1.5\%) withdrew consent, and 35 $(1.5 \%)$ were lost to follow-up.

\section{LABORATORY TESTING}

The mean LVEF for the entire study population was $24.7 \pm 7.5 \%$, with no significant difference between the warfarin and aspirin groups. Echocardiographic studies from 1854 of the 2305 patients in the study population $(80.4 \%)$ were analyzed at the core echocardiography laboratories; 1746 of these patients $(94.2 \%)$ had an LVEF of $35 \%$ or less or a wall-motion index of 1.2 or less. Baseline contrast angiography, radionuclide scanning, or MRI confirmed the eligibility of 239 of the 2305 patients (10.4\%), and the remaining 212 patients $(9.2 \%)$ entered the study with echocardiographic confirmation of LVEF or wallmotion-index values at the local site, without the core laboratory review.

After a 6-week period of dose adjustment, patients in the warfarin group had an INR in the therapeutic range, defined as 2.0 to 3.5 , for $62.6 \%$ of the follow-up time, as calculated with the use of a modification of the method of Rosendaal. ${ }^{19}$ INR values were below 2.0 for $27.1 \%$ of the total treatment time and above 3.5 for $10.3 \%$ of the total treatment time. In the warfarin group, the mean INR value during treatment was $2.5 \pm 0.95$.

\section{OUTCOMES}

Overall, 622 of the 2305 patients $(27.0 \%)$ had a primary outcome (531 deaths [85.4\%], 84 ischemic strokes [13.5\%], and 7 intracerebral hemorrhages [1.1\%]) (Table 2). The rates of the primary outcome were 7.47 events per 100 patient-years in the warfarin group and 7.93 per 100 patient-years in the aspirin group, with no significant difference between the two groups (hazard ratio with warfarin, $0.93 ; 95 \%$ confidence interval $[\mathrm{CI}], 0.79$ to $1.10 ; \mathrm{P}=$ 0.40) (Fig. 1). A time-varying analysis with the use of a Cox model showed a small benefit of warfarin as compared with aspirin over time. The hazard ratio decreased by a factor of 0.89 per year $(95 \% \mathrm{CI}, 0.80$ to $0.998 ; \mathrm{P}=0.046$ ) and became borderline significant by year 4 (hazard ratio with warfarin, $0.76 ; \mathrm{P}=0.04$ ) (Fig. 2).

In the entire patient population, there was a constant and significant benefit with warfarin as compared with aspirin with respect to the rate of ischemic stroke (hazard ratio, $0.52 ; 95 \%$ $\mathrm{CI}, 0.33$ to $0.82 ; \mathrm{P}=0.005$ ) (Table 2 ). The two treatment groups did not differ significantly with respect to the rate of intracerebral hemorrhage. Patients in the warfarin group did not receive the randomly assigned medication (and instead received open-label therapy) for 34\% of the total follow-up time, and patients in the aspirin group did not receive the assigned medication for $32 \%$ of the time. With respect to the main secondary outcome (first event in the composite of death, ischemic stroke, intracerebral hemorrhage, myocardial infarction, or hospitalization for heart failure), there was no significant difference between the warfarin group and the aspirin group (hazard ratio with warfarin, 1.07; 95\% CI, 0.93 to $1.23 ; \mathrm{P}=$ 0.33). The rates of myocardial infarction and hospitalization for heart failure did not differ significantly between the two groups, although there was a trend toward a higher rate of hospitalization for heart failure in the warfarin group $(\mathrm{P}=0.053)$ (Table 2). 
The rate of major hemorrhage was significantly higher with warfarin than with aspirin (1.78 events per 100 patient-years with warfarin vs. 0.87 per 100 patient-years with aspirin; adjusted rate ratio, 2.05; 95\% CI, 1.36 to 3.12; $\mathrm{P}<0.001$ ) (Table 3). However, the rates of intracerebral and intracranial hemorrhages combined did not differ significantly according to treatment group $(0.27$ events per 100 patient-years in the warfarin group and 0.22 per 100 patient-years in the aspirin group, $\mathrm{P}=0.82$ ). Major gastrointestinal bleeding occurred more frequently in the warfarin group $(0.94$ events per 100 patient-years vs. 0.45 per 100 patientyears in the aspirin group, $\mathrm{P}=0.01$ ). Table $\mathrm{S} 1$ in the Supplementary Appendix (available at NEJM.org) shows the most frequent and the most clinically relevant serious adverse events according to treatment group.

\section{DISCUSSION}

The WARCEF trial was designed to determine whether warfarin or aspirin is a better treatment for patients with a reduced LVEF who are in sinus rhythm. Previous studies either were retrospective or lacked the power to adequately address this issue. As a result, there has been insufficient evidence to support any strong treatment recommendations regarding the use of warfarin or aspirin in these patients. Our trial had a double-blind design with sham INRs, similar to that used in the Warfarin-Aspirin Recurrent Stroke Study (WARSS, NCT00027066), and used centralized INR processing centers to ensure that the INR data would be of high quality. ${ }^{18,20}$

Our results show no significant overall difference between warfarin and aspirin therapies in preventing the primary outcome. Although there may have been a small benefit with warfarin among patients followed for 4 or more years, it was of borderline statistical significance and uncertain clinical significance. There was a consistent and significant benefit of warfarin as compared with aspirin with respect to the prevention of ischemic stroke throughout the follow-up period. This benefit was suggested in the WATCH trial and has now been confirmed in the WARCEF trial, which included more patients and a longer follow-up period. ${ }^{16}$ However, the benefit was offset by the increase in the incidence of major bleeding. The relative reduction in the risk of ischemic stroke with warfarin among the patients in our study, who had heart failure, is similar to that observed among patients with atrial fibrillation. ${ }^{21}$ However, the absolute risk of ischemic stroke among patients with a low LVEF who are in sinus rhythm is significantly lower than that among patients with atrial fibrillation. ${ }^{16}$

With respect to the main secondary outcome, which included myocardial infarction and hospitalization for heart failure, in addition to the primary outcome, there was no significant difference between the warfarin group and the aspirin group. There was a trend toward an increased rate of hospitalization for heart failure in the warfarin group, a finding that is in direct contrast to the results of the WASH and WATCH trials, which suggested an increased rate of hospitalization for heart failure among patients receiving aspirin. ${ }^{15,16}$ There has been speculation that aspirin may in- terfere with prostaglandin synthesis, leading to a reduced effectiveness of ACE inhibition. ${ }^{22,23}$ In our trial, however, no increase in the rate of hospitalization for heart failure was seen in the aspirin group as compared with the warfarin group, even though a large proportion of patients in the aspirin group were treated with an ACE inhibitor.

In the warfarin group, the INR was in the therapeutic range of 2.0 to 3.5 for $63 \%$ of the total treatment time. We set the INR target above that used in trials involving patients with atrial fibrillation, because among trials involving patients who had had a myocardial infarction, those with higher INR targets and values showed the superiority of warfarin over aspirin, whereas those with lower INR targets and values did not. ${ }^{24,25}$ In our study, patients received 
either warfarin or aspirin and did not take both medications. The side-effect profile in the case of both warfarin and aspirin was generally acceptable, and there was a low rate of intracerebral hemorrhage. The rate of major hemorrhage was significantly increased with warfarin therapy but was lower than that seen in the warfarin group in recent trials involving patients with atrial fibrillation and similar to that seen in the WARSS and WATCH trials. ${ }^{16,20,26,27}$

The limitations of our study include the smaller-than-anticipated number of patients enrolled, and, given the variable length of follow-up, the relatively small numbers of patients who were still being followed in years 5 and 6 . The time in the therapeutic range among patients in the warfarin group was relatively low at $63 \%$. In addition, in both groups, there was a substantial portion of follow-up time during which the patients did not receive the assigned study treatment. However, this duration was similar in the two treatment groups, thus minimizing any bias. Since newer antithrombotic agents, as compared with warfarin, are easier to administer and may be associated with better long-term adherence to therapy, they may increase the time in the therapeutic range and reduce the time during which patients do not receive the assigned therapy. ${ }^{26-28}$ If so, they may prove to be more effective than warfarin or aspirin.

In summary, this trial showed no significant overall difference between warfarin and aspirin with respect to the primary outcome of death, ischemic stroke, or intracerebral hemorrhage. However, among patients followed for 4 or more years, there may have been a small benefit, of uncertain clinical significance, with warfarin. Warfarin was associated with a reduction in the risk of ischemic stroke throughout the follow-up period. Given the finding that warfarin did not provide an overall benefit and was associated with an increased risk of bleeding, there is no compelling reason to use warfarin rather than aspirin in patients with a reduced LVEF who are in sinus rhythm.

\title{
Supplementary Material
}

Refer to Web version on PubMed Central for supplementary material.

\section{Acknowledgments}

Supported by grants (U01-NS-043975 [to Dr. Homma] and U01-NS-039143 [to Dr. Thompson]) from the National Institute of Neurological Disorders and Stroke.

\begin{abstract}
Dr. Homma reports receiving payment from AGA Medical (now St. Jude Medical) for his work as a member of a data and safety monitoring board and consulting fees from Boehringer Ingelheim; Dr. Levin, receiving consulting fees from United Healthcare; Dr. Teerlink, receiving consulting fees from Amgen and grant support from Amgen, Cytokinetics, and Novartis on behalf of himself and from NovaCardia/Merck on behalf of himself and his institution; Dr. Graham, owning stock in March Pharmaceuticals, Medtronic, and Pfizer; Dr. Labovitz, receiving grant support from Boehringer Ingelheim on behalf of his institution, lecture fees from Boehringer Ingelheim, and fees for the development of educational presentations from the American College of Cardiology; Dr. Anker, receiving consulting fees from Amgen, Bosch Healthcare, GlaxoSmithKline, Helsinn, LoneStar Heart, Novartis, Professional Dietetics, PsiOxus, Relypsa, SHL Telemedicine, and Thermo Fisher, grant support from Vifor Pharma, and lecture fees from Novartis, holding patents with Brahms AG and Charité Berlin, and receiving royalties from Imperial College; Dr. Ponikowski, receiving consulting fees from Bayer, Boehringer Ingelheim, Coridea, Corthera, Johnson \& Johnson, Pfizer, Respicardia, and Vifor Pharma, grant support from Vifor Pharma on behalf of himself and his institution, and lecture fees from Abbott, Boehringer Ingelheim, Merck Serono, Pfizer, Respicardia, SanofiAventis, Servier, and Vifor Pharma; and Dr. Lip, receiving consulting fees from Astellas, AstraZeneca, Bayer, Biotronik, Boehringer Inhelheim, Bristol-Myers Squibb, Pfizer, Merck, Portola, and Sanofi-Aventis, speakers bureau fees from Bayer, Bristol-Myers Squibb, Pfizer, Boehringer Ingelheim, and Sanofi-Aventis, and payment for the development of educational presentations from Bayer, Boehringer Ingelheim, and Merck.
\end{abstract}

(Funded by the National Institute of Neurological Disorders and Stroke; WARCEF ClinicalTrials.gov number, NCT00041938.) 


\section{REFERENCES}

1. Kalaria VG, Passannante MR, Shah T, Modi K, Weisse AB. Effect of mitral regurgitation on left ventricular thrombus formation in dilated cardiomyopathy. Am Heart J. 1998; 135:215-220. [PubMed: 9489967]

2. Lip GYH, Gibbs CR. Does heart failure confer a hypercoagulable state? Virchow's triad revisited. J Am Coll Cardiol. 1999; 33:1424-1426. [PubMed: 10193748]

3. Uretsky BF, Thygesen K, Armstrong PW, et al. Acute coronary findings at autopsy in heart failure patients with sudden death: results from the Assessment of Treatment With Lisinopril and Survival (ATLAS) trial. Circulation. 2000; 102:611-616. [PubMed: 10931799]

4. Freudenberger RS, Halperin JL. Should we use anticoagulation for patients with chronic heart failure? Nat Clin Pract Cardiovasc Med. 2006; 3:580-581. [PubMed: 17063158]

5. Ezekowitz M. Antithrombotics for leftventricular impairment? Lancet. 1998; 351:1904. [PubMed: 9654255]

6. Sacco RL, Adams R, Albers G, et al. Guidelines for prevention of stroke in patients with ischemic stroke or transient ischemic attack: a statement for healthcare professionals from the American Heart Association/American Stroke Association Council on Stroke: co-sponsored by the Council on Cardiovascular Radiology and Intervention: the American Academy of Neurology affirms the value of this guideline. Circulation. 2006; 113(10):e409-e449. [PubMed: 16534023]

7. Anderson GM, Hull E. The effects of dicumarol upon the mortality and incidence of thromboembolic complications in congestive heart failure. Am Heart J. 1950; 39:697-702. [PubMed: 15413578]

8. Griffith GC, Stragnell R, Levinson DC, Moore FJ, Ware AG. A study of the beneficial effects of anticoagulant therapy in congestive heart failure. Ann Intern Med. 1952; 37:867-887. [PubMed: 12986600]

9. Harvey WP, Finch CA. Dicumarol prophylaxis of thromboembolic disease in congestive heart failure. N Engl J Med. 1950; 242:208-211. [PubMed: 15403339]

10. Al-Khadra AS, Salem DN, Rand WM, Udelson JE, Smith JJ, Konstam MA. Warfarin anticoagulation and survival: a cohort analysis from the Studies of Left Ventricular Dysfunction. J Am Coll Cardiol. 1998; 31:749-753. [PubMed: 9525542]

11. Loh E, Sutton MS, Wun CC, et al. Ventricular dysfunction and the risk of stroke after myocardial infarction. N Engl J Med. 1997; 336:251-257. [PubMed: 8995087]

12. Dunkman WB, Johnson GR, Carson PE, Bhat G, Farrell L, Cohn JN. Incidence of thromboembolic events in congestive heart failure. Circulation. 1993; 87:VI94-V101. [PubMed: 8500246]

13. Freudenberger RS, Hellkamp AS, Halperin JL, et al. Risk of thromboembolism in heart failure: an analysis from the Sudden Cardiac Death in Heart Failure Trial (SCD-HeFT). Circulation. 2007; 115:2637-2641. [PubMed: 17485579]

14. Cokkinos DV, Haralabopoulos GC, Kostis JB, Toutozas PK. Efficacy of antithrombotic therapy in chronic heart failure: the HELAS study. Eur J Heart Fail. 2006; 8:428-432. [PubMed: 16737850]

15. Cleland JGF, Findlay I, Jafri S, et al. The Warfarin/Aspirin Study in Heart Failure (WASH): a randomized trial comparing antithrombotic strategies for patients with heart failure. Am Heart J. 2004; 148:157-164. [PubMed: 15215806]

16. Massie BM, Collins JF, Ammon SE, et al. Randomized trial of warfarin, aspirin, and clopidogrel in patients with chronic heart failure: the Warfarin and Antiplatelet Therapy in Chronic Heart Failure (WATCH) trial. Circulation. 2009; 119:1616-1624. [PubMed: 19289640]

17. Pullicino $\mathrm{P}$, Thompson JL, Barton B, et al. Warfarin versus aspirin in patients with reduced cardiac ejection fraction (WARCEF): rationale, objectives, and design. J Card Fail. 2006; 12:39-46. [PubMed: 16500579]

18. Thompson JLP, Fleiss JL, James K, et al. Test of an algorithm for simulating prothrombin times in a double-blind anticoagulation drug trial. Ann Neurol. 1994; 36:305-306. abstract.

19. Rosendaal FR, Cannegieter SC, van der Meer FJ, Briët E. A method to determine the optimal intensity of oral anticoagulant therapy. Thromb Haemost. 1993; 69:236-239. [PubMed: 8470047]

20. Mohr JP, Thompson JL, Lazar RM, et al. A comparison of warfarin and aspirin for the prevention of recurrent ischemic stroke. N Engl J Med. 2001; 345:1444-1451. [PubMed: 11794192] 
21. Hart RG, Benavente O, McBride R, Pearce LA. Antithrombotic therapy to prevent stroke in patients with atrial fibrillation: a meta-analysis. Ann Intern Med. 1999; 131:492-501. [PubMed: 10507957]

22. Hall D, Zeitler H, Rudolph W. Counteraction of the vasodilator effects of enalapril by aspirin in severe heart failure. J Am Coll Cardiol. 1992; 20:1549-1555. [PubMed: 1452929]

23. Spaulding C, Charnonnier B, Cohen-Solal A, et al. Acute hemodynamic interaction of aspirin and ticlopidine with enalapril: results of a double-blind, randomized comparative trial. Circulation. 1998; 98:757-765. [PubMed: 9727545]

24. Hurlen M, Abdelnoor M, Smith P, Erikssen J, Arnesen H. Warfarin, aspirin, or both after myocardial infarction. N Engl J Med. 2002; 347:969-974. [PubMed: 12324552]

25. van Es RF, Jonker JJ, Verheugt FW, Deckers JW, Grobbee DE. Aspirin and Coumadin After Acute Coronary Syndromes (the ASPECT-2 study): a randomized controlled trial. Lancet. 2002; 360:109-113. [PubMed: 12126819]

26. Connolly SJ, Ezekowitz MD, Yusuf S, et al. Dabigatran versus warfarin in patients with atrial fibrillation. N Engl J Med. 2009; 361:1139-1151. [Erratum, N Engl J Med 2010;363:1877.]. [PubMed: 19717844]

27. Patel MR, Mahaffey KW, Garg J, et al. Rivaroxaban versus warfarin in nonvalvular atrial fibrillation. N Engl J Med. 2011; 365:883-891. [PubMed: 21830957]

28. Granger CB, Alexander JH, McMurray JJV, et al. Apixaban versus warfarin in patients with atrial fibrillation. N Engl J Med. 2011; 365:981-992. [PubMed: 21870978] 


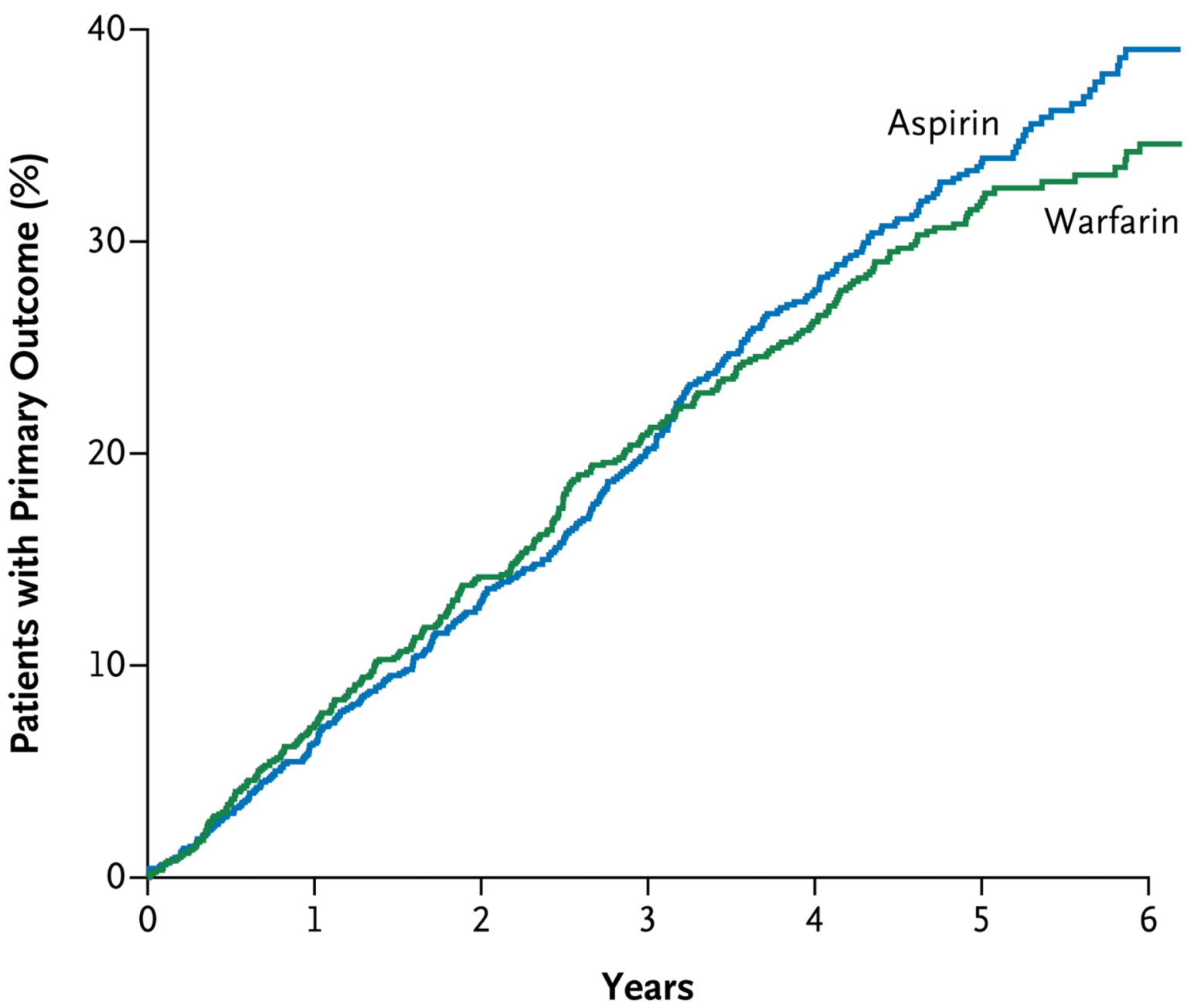

No. at Risk

Aspirin Warfarin
1163

1142
1073

1049
860

852
658

653
508

525

Figure 1. Cumulative Incidence of the Primary Outcome

The primary outcome was the time to the first event in the composite end point of ischemic stroke, intracerebral hemorrhage, or death from any cause. 


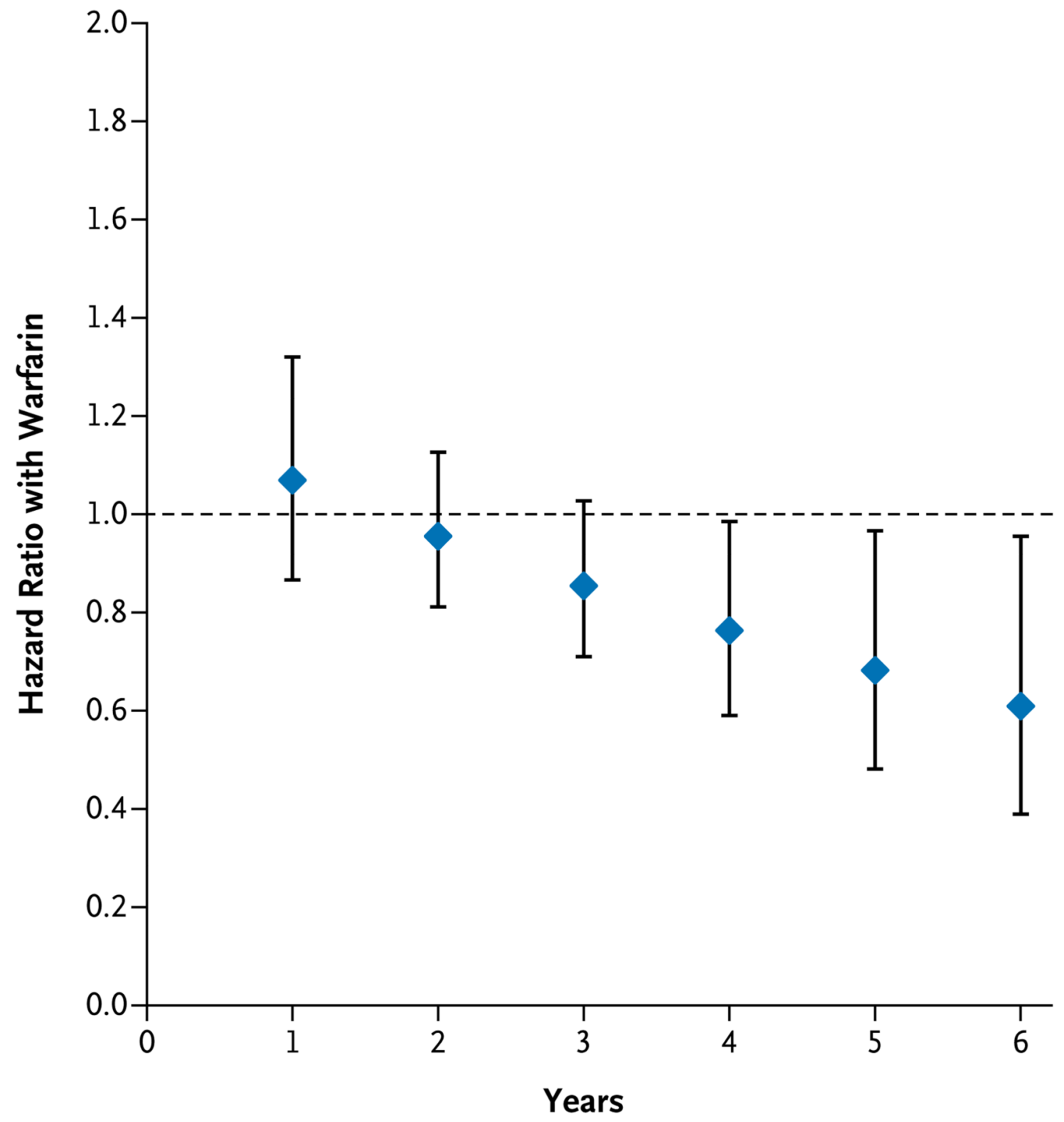

No. at Risk

Aspirin

Figure 2. Hazard Ratios for the Primary Outcome with Warfarin, According to Year of Followup

Hazard ratios were estimated with the use of a stratified Cox model that expressed the logrelative hazard ratio as a linear function of follow-up time. The hazard ratio decreased by a factor of 0.89 per year $(95 \%$ confidence interval, 0.80 to $0.998 ; \mathrm{P}=0.046)$. I bars indicate $95 \%$ confidence intervals. 


\section{Table 1}

Baseline Characteristics of the Study Participants, According to Treatment Group. *

\begin{tabular}{|c|c|c|}
\hline Characteristic & Warfarin $(N=1142)$ & Aspirin $(\mathrm{N}=1163)$ \\
\hline Age - yr & $61 \pm 11.6$ & $61 \pm 11.1$ \\
\hline \multicolumn{3}{|l|}{ Location - no. $(\%)$} \\
\hline North America & $573(50.2)$ & $546(46.9)$ \\
\hline Europe & $527(46.1)$ & $567(48.8)$ \\
\hline Argentina & $42(3.7)$ & $50(4.3)$ \\
\hline Male sex — no./total no. (\%) & $904 / 1140(79.3)$ & $936 / 1160(80.7)$ \\
\hline \multicolumn{3}{|l|}{ Race or ethnic group - no./total no. $(\%)^{\dagger}$} \\
\hline Non-Hispanic white & $857 / 1140(75.2)$ & $876 / 1159(75.6)$ \\
\hline Non-Hispanic black & $166 / 1140(14.6)$ & $166 / 1159(14.3)$ \\
\hline Hispanic & $85 / 1140(7.5)$ & $81 / 1159(7.0)$ \\
\hline Other & $32 / 1140(2.8)$ & $36 / 1159(3.1)$ \\
\hline Height $-\mathrm{cm}$ & $172 \pm 9.3$ & $172 \pm 9.2$ \\
\hline Weight $-\mathrm{kg}$ & $86 \pm 19.6$ & $87 \pm 19.3$ \\
\hline \multicolumn{3}{|l|}{ Body-mass index ${ }^{t}$} \\
\hline Mean & $29 \pm 5.9$ & $29 \pm 6$ \\
\hline \multicolumn{3}{|l|}{ Distribution — no./total no. (\%) } \\
\hline$<25$ & 294/1135 (25.9) & $265 / 1149(23.1)$ \\
\hline $25-30$ & $426 / 1135(37.5)$ & $456 / 1149(39.7)$ \\
\hline$>30$ & $415 / 1135(36.6)$ & $428 / 1149(37.2)$ \\
\hline \multicolumn{3}{|l|}{ Blood pressure $-\mathrm{mm} \mathrm{Hg}$} \\
\hline Systolic & $124 \pm 19.3$ & $124 \pm 18.4$ \\
\hline Diastolic & $74 \pm 11.6$ & $74 \pm 11.3$ \\
\hline Pulse - beats/min & $72 \pm 11.4$ & $72 \pm 12.5$ \\
\hline Hypertension - no./total no. (\%) & $671 / 1104(60.8)$ & $696 / 1128(61.7)$ \\
\hline Diabetes mellitus - no./total no. $(\%)$ & $371 / 1138(32.6)$ & $351 / 1156(30.4)$ \\
\hline Atrial fibrillation — no./total no. $(\%)$ & $44 / 1139(3.9)$ & $42 / 1156(3.6)$ \\
\hline Myocardial infarction - no./total no. (\%) & $549 / 1138(48.2)$ & $563 / 1156(48.7)$ \\
\hline Ischemic cardiomyopathy — no./total no. (\%) & $488 / 1138(42.9)$ & $503 / 1155(43.5)$ \\
\hline Pulmonary or other embolism — no./total no. (\%) & $28 / 1139(2.5)$ & $24 / 1155(2.1)$ \\
\hline \multicolumn{3}{|l|}{ Smoking status - no./total no. (\%) } \\
\hline Current smoker & $213 / 1138(18.7)$ & $195 / 1158(16.8)$ \\
\hline Former smoker & $581 / 1138(51.1)$ & $599 / 1158(51.7)$ \\
\hline Never smoked & $344 / 1138(30.2)$ & $364 / 1158(31.4)$ \\
\hline \multicolumn{3}{|l|}{ Alcohol consumption - no./total no. (\%) } \\
\hline Current consumption, $>2$ oz/day & $279 / 1140(24.5)$ & $293 / 1158(25.3)$ \\
\hline Previous consumption, $>2 \mathrm{oz} /$ day & $250 / 1140(21.9)$ & $256 / 1158(22.1)$ \\
\hline
\end{tabular}




\begin{tabular}{|c|c|c|}
\hline Characteristic & Warfarin $(N=1142)$ & Aspirin $(\mathrm{N}=1163)$ \\
\hline Never consumed alcohol & $611 / 1140(53.6)$ & $609 / 1158(52.6)$ \\
\hline \multicolumn{3}{|l|}{ Educational level — no./total no. (\%) } \\
\hline$<$ High school & $490 / 1140(43.0)$ & $502 / 1155(43.5)$ \\
\hline High-school graduate or some college & $487 / 1140(42.7)$ & $460 / 1155(39.8)$ \\
\hline College graduate or postgraduate & $163 / 1140(14.3)$ & $193 / 1155(16.7)$ \\
\hline \multicolumn{3}{|l|}{ NYHA classification — no./total no. $(\%)^{\xi}$} \\
\hline $\mathrm{I}$ & $150 / 1137(13.2)$ & $165 / 1153(14.3)$ \\
\hline II & $621 / 1137(54.6)$ & $646 / 1153(56.0)$ \\
\hline III & $351 / 1137(30.9)$ & $329 / 1153(28.5)$ \\
\hline IV & $15 / 1137(1.3)$ & $13 / 1153(1.1)$ \\
\hline Ejection fraction $-\%$ & $25 \pm 7.5$ & $25 \pm 7.5$ \\
\hline Distance covered on 6-minute walk $-\mathrm{m}^{q /}$ & $346 \pm 147.3$ & $356 \pm 152.5$ \\
\hline Prior stroke or TIA — no./total no. (\%) & $155 / 1138(13.6)$ & $139 / 1157(12.0)$ \\
\hline \multicolumn{3}{|c|}{ Score on modified Rankin scale - no./total no. $(\%)^{/ /}$} \\
\hline \multicolumn{3}{|l|}{ All patients } \\
\hline 0 & $463 / 1133(40.9)$ & $489 / 1157(42.3)$ \\
\hline 1 & $353 / 1133(31.2)$ & $359 / 1157(31.0)$ \\
\hline 2 & $262 / 1133(23.1)$ & $266 / 1157(23.0)$ \\
\hline 3 & $46 / 1133(4.1)$ & $40 / 1157(3.5)$ \\
\hline 4 & $9 / 1133(0.8)$ & $3 / 1157(0.3)$ \\
\hline \multicolumn{3}{|l|}{ Patients with prior stroke or TIA } \\
\hline 0 & $40 / 154(26.0)$ & $38 / 139(27.3)$ \\
\hline 1 & $50 / 154(32.5)$ & $43 / 139(30.9)$ \\
\hline 2 & $48 / 154(31.2)$ & $48 / 139(34.5)$ \\
\hline 3 & $12 / 154(7.8)$ & $9 / 139(6.5)$ \\
\hline 4 & $4 / 154(2.6)$ & $1 / 139(0.7)$ \\
\hline \multicolumn{3}{|l|}{ Medications - no./total no. $(\%)^{* *}$} \\
\hline Aspirin ${ }^{\dagger t}$ & $611 / 1047(58.4)$ & $632 / 1071(59.0)$ \\
\hline Other antiplatelet agent ${ }^{\dagger \dagger}$ & $32 / 428(7.5)$ & $40 / 461(8.7)$ \\
\hline Warfarin or other oral anticoagulant $t^{\dagger}$ & $90 / 1142(7.9)$ & $89 / 1163(7.7)$ \\
\hline ACE inhibitor or ARB & $1118 / 1136(98.4)$ & $1139 / 1157(98.4)$ \\
\hline Beta-blocker & $1026 / 1136(90.3)$ & $1036 / 1158(89.5)$ \\
\hline Aldosterone blocker & $406 / 666(61.0)$ & $407 / 679(59.9)$ \\
\hline Nitrate & $284 / 1135(25.0)$ & $259 / 1158(22.4)$ \\
\hline Calcium-channel blocker & $100 / 1135(8.8)$ & $103 / 1156(8.9)$ \\
\hline Diuretic & $925 / 1136(81.4)$ & $930 / 1158(80.3)$ \\
\hline Statin & $690 / 827(83.4)$ & $704 / 851(82.7)$ \\
\hline Device - no./total no. (\%) & & \\
\hline
\end{tabular}




\begin{tabular}{|lcc|}
\hline Characteristic & Warfarin $(\mathbf{N}=\mathbf{1 1 4 2})$ & Aspirin $(\mathbf{N}=\mathbf{1 1 6 3})$ \\
\hline Pacemaker & $141 / 1139(12.4)$ & $144 / 1156(12.5)$ \\
\hline Implantable cardioverter-defibrillator & $212 / 1139(18.6)$ & $206 / 1156(17.8)$ \\
\hline
\end{tabular}

Plus-minus values are means \pm SD. None of the differences between the warfarin group and the aspirin group were significant (P>0.05). Continuous variables were compared with the use of Student's t-test. Binary categorical variables were compared with the use of Fisher's exact test, and multicategory variables were compared with the use of standard chi-square tests. ACE denotes angiotensin-converting enzyme, ARB angiotensin-receptor blocker, and TIA transient ischemic attack.

${ }^{\dagger}$ Race and ethnic group were self-reported separately and were combined for presentation.

The body-mass index is the weight in kilograms divided by the square of height in meters.

$\mathcal{S}_{\text {The New York Heart Association (NYHA) classification groups patients with heart failure according to the extent of limitation during physical }}$ activity. Class I indicates no limitation, and class IV severe limitation.

II Data on the distance covered on a 6-minute walk were available for 2102 (1031 in the warfarin group and 1071 in the aspirin group) of the 2305 patients $(91.2 \%)$.

"Scores on the modified Rankin scale range from 0 to 6 , with 0 indicating no symptoms and 6 indicating death. In this study, the scores ranged from 0 to 4 ; a score higher than 4 was a protocol-specified criterion for exclusion.

***

Data on medications were obtained from the case-report form at the screening visit.

${ }^{\dagger}+$ underwent randomization. 


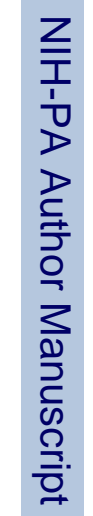

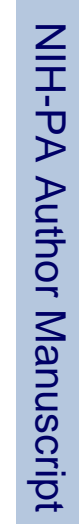

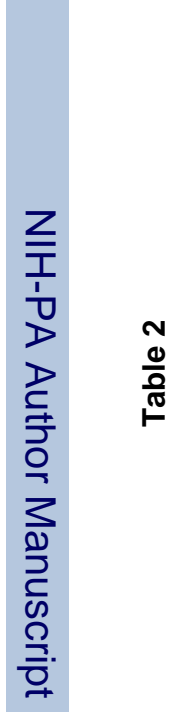

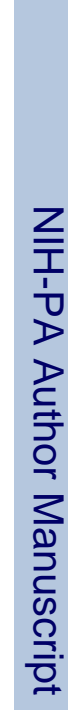

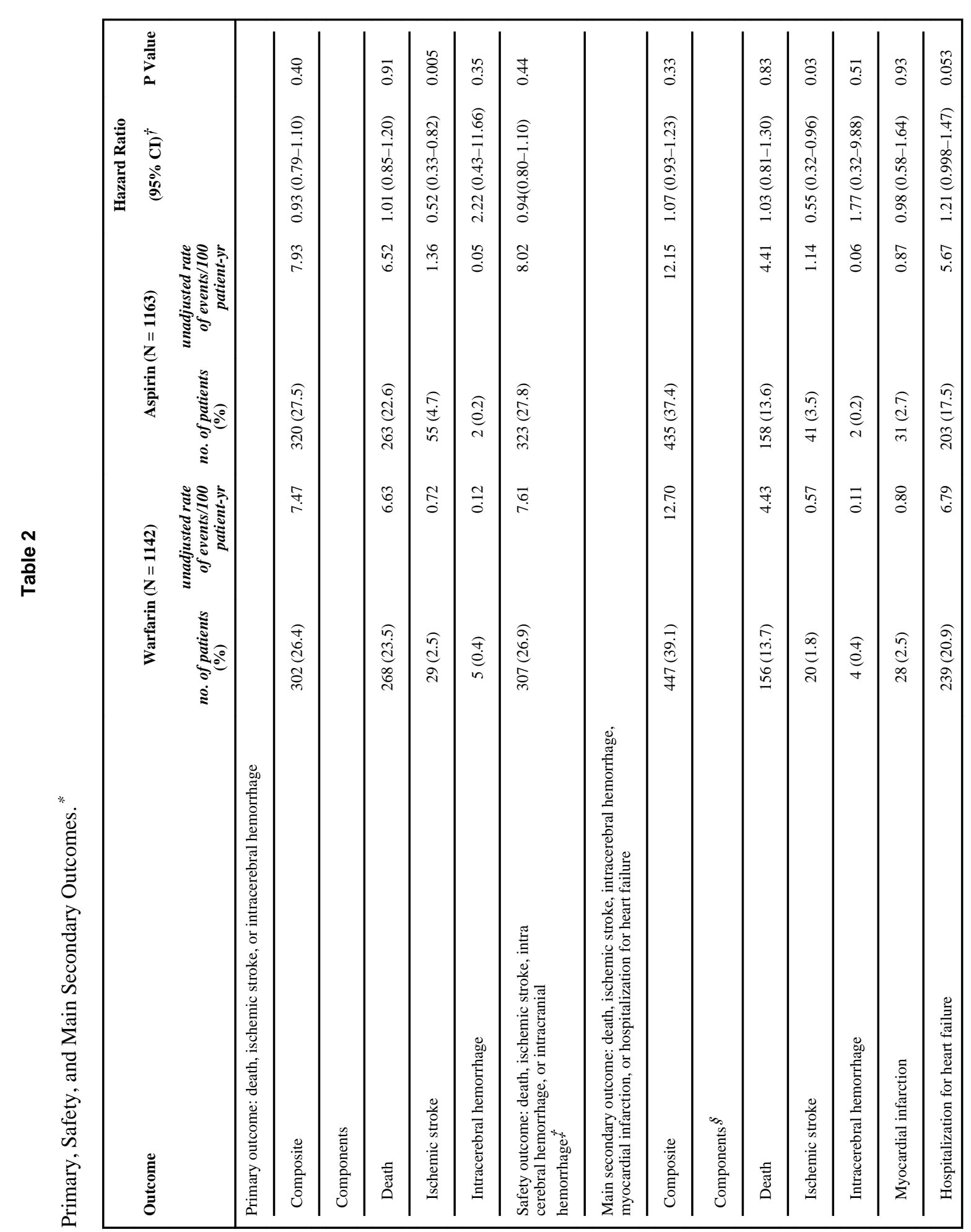

N Engl J Med. Author manuscript; available in PMC 2013 July 25. 


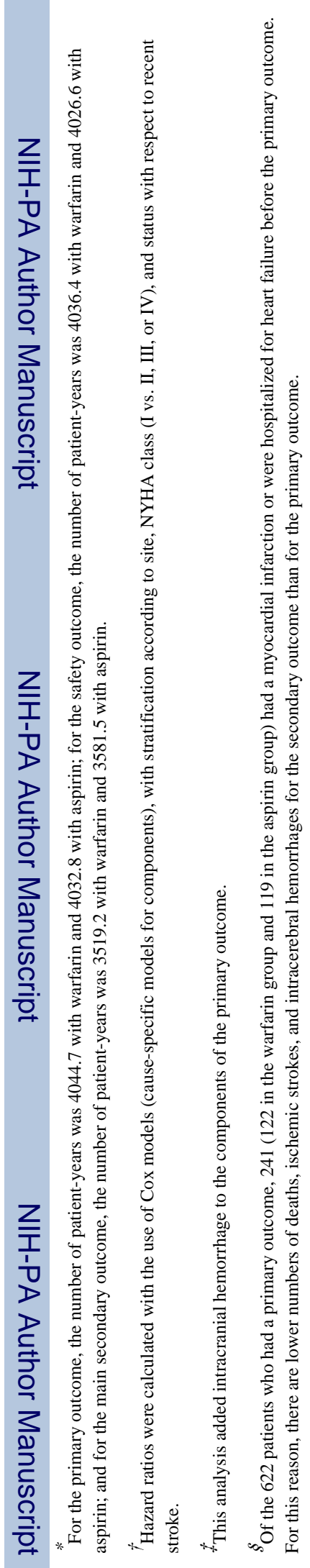

N Engl J Med. Author manuscript; available in PMC 2013 July 25. 
Table 3

Rates of Hemorrhage and Death, According to Treatment Group. *

\begin{tabular}{|c|c|c|c|c|}
\hline Event & Warfarin $(N=1142)$ & Aspirin $(\mathrm{N}=1163)$ & $\begin{array}{l}\text { Odds Ratio or Rate } \\
\text { Ratio }(95 \% \text { CI })^{\dagger}\end{array}$ & P Value \\
\hline \multicolumn{5}{|l|}{ Death as part of primary outcome - no. of patients (\%) } \\
\hline From any cause & $268(23.5)$ & $263(22.6)$ & $1.05(0.86-1.27)$ & 0.66 \\
\hline Related to hemorrhage ${ }^{\xi}$ & $7(0.6)$ & $4(0.3)$ & $1.84(0.54-6.32)$ & 0.38 \\
\hline \multicolumn{5}{|l|}{ Death after primary outcome - no. of patients $(\%) q l$} \\
\hline After ischemic stroke & $5(0.4)$ & $7(0.6)$ & $0.71(0.22-2.40)$ & 0.77 \\
\hline After intracerebral hemorrhage & $2(0.2)$ & $2(0.2)$ & $0.98(0.11-9.10)$ & 1.00 \\
\hline Major hemorrhage - no. of patients $(\%)^{/ /}$ & $66(5.8)$ & $31(2.7)$ & $2.21(1.42-3.47)$ & $<0.001$ \\
\hline Intracerebral & $5(0.4)$ & $2(0.2)$ & $2.52(0.52-17.9)$ & 0.29 \\
\hline Intracranial $^{* *}$ & $5(0.4)$ & $7(0.6)$ & $0.72(0.22-2.43)$ & 0.77 \\
\hline Gastrointestinal & $37(3.2)$ & $16(1.4)$ & $2.35(1.30-4.38)$ & 0.005 \\
\hline Other & $21(1.8)$ & $7(0.6)$ & $3.06(1.26-7.57)$ & 0.008 \\
\hline Minor hemorrhage — no. of patients (\%) & $280(24.5)$ & $189(16.3)$ & $1.65(1.34-2.05)$ & $<0.001$ \\
\hline \multicolumn{5}{|l|}{ All hemorrhages ${ }^{\dagger \dagger}$} \\
\hline Total no. of patient-yr & 4044.7 & 4032.8 & & \\
\hline Major hemorrhage - no. of events (no./100 patient-yr) & $72(1.78)$ & $35(0.87)$ & $2.05(1.36-3.12)$ & $<0.001$ \\
\hline Intracerebral & $5(0.12)$ & $2(0.05)$ & $2.48(0.51-17.6)$ & 0.45 \\
\hline Intracranial $^{* *}$ & $6(0.15)$ & $7(0.17)$ & $0.86(0.29-2.85)$ & 1.00 \\
\hline Gastrointestinal & $38(0.94)$ & $18(0.45)$ & $2.10(1.19-3.70)$ & 0.010 \\
\hline All other & $23(0.57)$ & $8(0.2)$ & $2.88(1.30-6.94)$ & 0.01 \\
\hline Minor hemorrhage - no. of events (no./100 patient-yr) & $468(11.6)$ & $296(7.34)$ & $1.56(1.34-1.81)$ & $<0.001$ \\
\hline
\end{tabular}

*

The maximum follow-up time was 74.3 months. Hemorrhages that occurred on the day of the primary event (death, ischemic stroke, or intracerebral hemorrhage) are included.

Odds ratios are shown for all categories with number and percent of patients; rate ratios are shown for all categories with number of events and rate per 100 person-years. Odds ratios and rate ratios are conditional maximum-likelihood estimates, stratified according to geographic location (North America, Europe, or Argentina). No test for heterogeneity of odds ratios or rate ratios across geographic locations was significant at the 0.05 level; the smallest $\mathrm{P}$ value for heterogeneity was 0.08 for the rate of minor hemorrhage.

${ }^{\mathrm{P}}$ values for categories with number and percent of patients were calculated with the use of the exact test of two independent proportions, stratified according to geographic location. $P$ values for categories with number of events and rate per 100 patient-years were calculated with the use of the exact conditional binomial test for two independent Poisson variables, stratified according to geographic location.

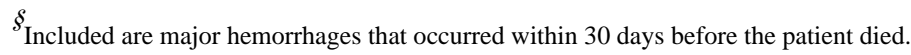

II These deaths are not primary end points and are not included in the total number of deaths in this table or in Table 2.

"Included is the first or only hemorrhage for each patient.

** Intracranial hemorrhages include intracranial or spinal hemorrhages, subarachnoid hemorrhages, subdural or epidural hemorrhages, and retinal hemorrhages.

${ }^{\dagger}$ Included are all hemorrhages that occurred in any patient. 